\title{
Prediction of ceramic stereolithography resin sensitivity from theory and measurement of diffusive photon transport
}

\author{
K. C. Wu \\ Department of Materials Science and Engineering, University of Michigan, Ann Arbor, Michigan 48109 \\ K. F. Seefeldt and M. J. Solomon \\ Department of Chemical Engineering, University of Michigan, Ann Arbor, Michigan 48109 \\ J. W. Halloran ${ }^{\text {a) }}$ \\ Department of Materials Science and Engineering, University of Michigan, Ann Arbor, Michigan 48109
}

(Received 4 November 2004; accepted 1 June 2005; published online 19 July 2005)

\begin{abstract}
A general, quantitative relationship between the photon-transport mean free path $\left(l^{*}\right)$ and resin sensitivity $\left(D_{P}\right)$ in multiple-scattering alumina/monomer suspensions formulated for ceramic stereolithography is presented and experimentally demonstrated. A Mie-theory-based computational method with structure factor contributions to determine $l^{*}$ was developed. Planar-source diffuse transmittance experiments were performed on monodisperse and bimodal polystyrene/water and alumina/monomer systems to validate this computational tool. The experimental data support the application of this $l^{*}$ calculation method to concentrated suspensions composed of nonaggregating particles of moderately aspherical shape and log-normal size distribution. The values of $D_{P}$ are shown to be approximately five times that of $l^{*}$ in the tested ceramic stereolithography suspensions. (C) 2005 American Institute of Physics. [DOI: 10.1063/1.1980531]
\end{abstract}

\section{INTRODUCTION}

Ceramic stereolithography is a solid free-form fabrication method capable of producing complex threedimensional (3D)-architectured ceramic green parts directly from computer-assisted design software. Adapted from commercially available stereolithography systems, computercontrolled lenses and mirrors guide a monochromatic ultraviolet laser beam $(325 \mathrm{~nm})$ onto the surface of an acrylate monomer suspension containing photoinitiators and approximately $50-$ vol \% nonabsorbing ceramic particles, enabling photopolymerization of successive two-dimensional (2D) patterns of specified thicknesses in the vertical direction. Each photopolymerized layer adheres to the previous layer beneath by an excess dose of energy (overcure), allowing the fabrication of complex 3D structures. ${ }^{1,2}$ Successful ceramic suspensions for stereolithography contain at least $50 \%$ loading by volume to ensure green body integrity while maintaining a "pourable" viscosity to aid in the recoating process. ${ }^{1,3}$

The critical parameters in stereolithography resins are resin sensitivity $\left(D_{P}\right)$, the distance at which the laser intensity is reduced by $e^{-1}$, and cure depth $\left(C_{D}\right)$, the distance of photopolymerization through the surface as specified by the user. They are related through the Jacobs equation [Eq. (1)], where $E_{\text {laser }}$ and $E_{C}$ are the energy $\left(\mathrm{mJ} / \mathrm{cm}^{2}\right)$ delivered to the surface of the resin and the critical energy of photopolymerization, respectively, ${ }^{2}$

$$
C_{D}=D_{P} \ln \frac{E_{\text {laser }}}{E_{C}} .
$$

However, the introduction of ceramic particles in these suspensions adds a level of complexity because laser illumination may be multiply scattered by the suspension instead

${ }^{a)}$ Electronic mail: peterjon@umich.edu of being absorbed by the photoinitiator. This situation reduces the cure depth and the overall build speed. The reason for the degradation in performance is that such colloidal systems strongly scatter light due to the difference in refractive index between the particles and the dispersing medium. In the limit of strong multiple scattering, recent studies have shown that light propagation can be modeled by means of the photon diffusion approximation. ${ }^{3-9}$ In this case, multiple scattering can be characterized by a transport mean free path $l^{*}$, the typical step length of the photon random walk. ${ }^{3,8,10,11}$ In a dilute suspension, these values are dependent on the number density of scatterers $\rho$, the scattering cross section $\sigma$, and an ensemble average of $(1-\cos \theta)$ over all possible scattering angles $(\theta)$ occurring between events, ${ }^{3,12}$

$$
l^{*}=\frac{(\rho \sigma)^{-1}}{\langle 1-\cos \theta\rangle} .
$$

Such a quantitative treatment of light scattering arises from generalized solutions of Maxwell's equations, in conjunction with the diffusive transport theory of photon propagation in multiple-scattering systems. The diffusion approximation describes the random-walk propagation of photons through a multiple-scattering sample between successive scattering events. Physically, these samples have an opaque or milklike appearance, and their propensity to scatter light is often characterized by opacity, defined to be $1 / l^{*}$. In the multiple-scattering limit, for which the path length $L$ is much greater than $l^{*}$, essentially none of the light traverses the path length unscattered so that the transmitted light is mostly uncorrelated to the incident beam.

Light-scattering techniques such as static light scattering (SLS), ${ }^{13}$ dynamic light scattering (DLS), ${ }^{14,15}$ diffusive wave spectroscopy (DWS), ${ }^{8,9,15-17}$ and diffusive transmittance spectroscopy $^{5,12}$ (DTS) are powerful tools to extract static 
and dynamic measures of colloidal suspensions, such as structure, scattering power, particle size, and Brownian motion. This information can then be fruitfully applied to technologies such as materials processing, pharmaceutical sciences, and medical imaging technology. ${ }^{4-6,16,18-20,29,30}$

Multiple scattering in situations of technological interest rarely results from dilute suspensions of monodisperse, spherical particles. Yet, early studies devoted to this subject provide insight necessary to apply scattering methods to the suspensions typical of technical applications. For example, Wyss et al., ${ }^{16}$ Kaplan et al., ${ }^{2}$ and Fraden and Maret ${ }^{18}$ observed the effect of interparticle correlations in concentrated, multiply scattering suspensions. Kaplan et al. showed the significance of interparticle interactions in predicting opacity in monodisperse and bimodal mixtures of spheres modeled as hard spheres. ${ }^{8}$ Kaplan et al. showed, by calculation and experimentation, that the addition of small spheres to a relatively dilute dispersion of large spheres resulted in a decrease in scattering. ${ }^{21}$ In binary systems, this effect was attributed to the cross-component structural interactions between large and small particles. ${ }^{10}$ Seefeldt and Solomon assessed the role of attractive interparticle potential interactions on colloid self-diffusion. $^{22}$

Scattering in dilute monodisperse, spherical particulate suspensions may be described by Mie scattering theory. ${ }^{11}$ In the dilute limit, $l^{*}$ should decrease linearly as the particle concentration increases. However, in more concentrated suspensions, the binary interparticle correlations play a significant role through the structure factor $S(q)$, the Fourier transform of the particulate radial distribution function. ${ }^{23}$ For correlated scatterers, the decrease in $l^{*}$ is less than linear; Fraden and Maret used scattering methods to confirm the deviations of opacity from linearity in suspensions with volume fraction $\phi$ ranging from 0.05 to $0.45 .{ }^{18}$ Garg et al. used a pulse transmission light-scattering experiment in highly concentrated suspensions to show the total photon attenuation length in ceramic stereolithography suspensions scaled as a function of the interparticle spacing. They also observed a deviation from linearity at high volume fractions where the correlation effects significantly decreased opacity in alumina particle suspensions above a critical volume fraction. ${ }^{3}$

These scattering analyses may form the basis of a quantitative predictive model relating $D_{P}$ in ceramic stereolithography to opacity. The $l^{*}$ parameter itself would be dependent on the physical characteristics of the ceramic of interest, namely, refractive index, particle size, size distribution, and interaction potential. This model can then be used by stereolithography practitioners to explore methods of controlling $D_{P}$ in ceramic suspensions and thereby provide advantages in build time and cost. Furthermore, knowledge of the scattering behavior may be useful in developing future tools to compensate for scattering-induced line resolution broadening.

This paper discusses a set of light-scattering experiments which were performed to optically characterize nonideal suspensions having size polydispersity, modest aspherical and nonuniform shape, and at higher $\phi$. These measurements were then used to validate a computation of opacity in concentrated, bimodal suspensions. The Mie-theory-based calcu- lation method provides values of opacity for comparison in each case given the input parameters of incident wavelength, refractive indices of the particle and the medium, particle size, and particle $\phi .{ }^{10}$ DTS experiments were performed with polystyrene colloidal suspensions in two types of suspensions: monodisperse suspensions of $\phi_{\text {total }}$ ranging from 0.1 to 0.6 and bimodal suspensions with varying $\phi_{\text {small }}$ at a fixed total volume fraction of 0.5 . These two kinds of suspensions are of particular interest because the data can be used to evaluate the validity of our computational model for opacity, which incorporates the structure factor effects in multiple light scattering. DTS experiments were also performed with alumina/monomer suspensions to test the predictive value of the calculation for particles of log-normal size distribution and modestly aspherical geometry. Good agreement between the calculated and experimental opacity values suggest the applicability of the calculation to determine opacity with a range of material refractive indices, particle sizes, and volume fractions in both monodisperse and bimodal suspensions.

With both theoretical and experimental measurements of opacity available and with direct measurement of $D_{P}$ possible, we then proceeded to study the relationship between these two quantities. We hypothesize that $D_{P}$ is related to optical properties of the suspension by

$$
\frac{1}{D_{P}(1-\phi) c_{\mathrm{PI}}} \approx A \frac{1}{l^{*}(1-\phi) c_{\mathrm{PI}}}+\alpha_{\mathrm{PI}},
$$

where $A$ is an empirical constant, $\phi$ is the particle volume fraction, $\alpha_{\mathrm{PI}}$ is the molar absorptivity $\left(\mathrm{M}^{-1} \mu \mathrm{m}^{-1}\right)$ of the UV photoinitiator, and $c_{\mathrm{PI}}$ is the concentration of the photoinitiator. This equation of linear form shows that $D_{P}$ changes with $l^{*}$, which depends upon particle volume fraction and particle size distribution. Equation (3) may facilitate optimization and tailoring of the ceramic stereolithography process since the optical properties of ingredients in the ceramic suspensions of interest are easily obtained.

\section{EXPERIMENT}

\section{A. Materials}

Three sizes of monodisperse polystyrene (Optibind) spheres, each with a manufacturer-reported maximum of 5\% size deviation, were obtained from Seradyn, Inc. (Indianapolis, IN). The mean radii of the particles were $0.152,0.260$, and $0.547 \mu \mathrm{m}$. The particles were synthesized by a free radical emulsion-polymerization of styrene. The polymer chains are terminated with a sulfate group. Particles were dispersed in de-ionized water to obtain the $\phi$ of interest. Higher volume fractions were obtained by repeated centrifugation and resuspension of the as-received 10- and 30-vol \% suspensions. The refractive index of the polystyrene spheres was reported to be 1.59 .

Alumina powders A1000SG, A16SG, and A3500SG were obtained from Alcoa (Point Comfort, TX), with mean particle radii of $0.150,0.200$, and $1.50 \mu \mathrm{m}$, respectively. The particle size distributions were measured with a Horiba CAPA 700 particle size analyzer and are reported in Fig. 1, indicating log-normal size dispersion. Prior to use, the pow- 


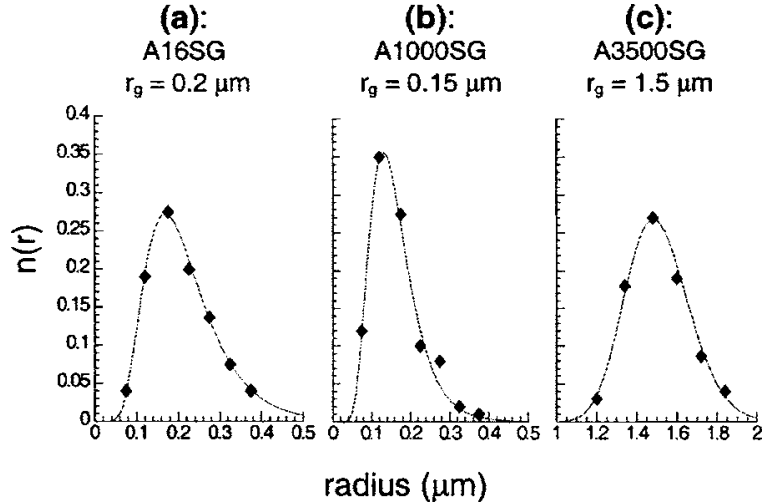

FIG. 1. Measured size distribution of particles (points) with fitted lognormal distribution functions.

ders were stored in a drying oven to minimize moistureinduced particle agglomeration. Suspensions of various loadings were prepared in a low-viscosity monomer, 1,6hexanediol diacrylate (HDDA) (SR238, $M_{W}=240 \mathrm{~g} / \mathrm{mol}$ ), obtained from Sartomer (Exton, PA), with a measured refractive index of 1.456. To aid in suspension processing, $2 \%$ dry-weight basis of an ammonium phosphate dispersant, with a measured refractive index of 1.44 , was added. Alumina suspensions were prepared by incrementally mixing solid and liquid components and ball milling for at least $48 \mathrm{~h}$ with $3 / 8$-in. alumina milling media. However, in the alumina transmittance experiments at total volume loadings below $20 \%$, the particles tended to sediment. In this case a tetraacrylate monomer (ethoxylated pentaerythritol tetraacrylate, SR494) with a higher viscosity of $108 \mathrm{mPa}$ s, was used to create a more stable dispersion. The refractive index of the tetraacrylate monomer $(n=1.470)$ did not differ too greatly from the diacrylate $(n=1.456)$ and the effect of this change is accounted for in comparison to theory. The photoinitiator added to the alumina/monomer suspensions was 1-benzoyl1-hydroxycyclohexane (Irgacure 184), obtained from Ciba SC (Tarrytown, NY), with a molar extinction coefficient of $700 \mathrm{M}^{-1} \mathrm{~cm}^{-1}$ at a wavelength of $325 \mathrm{~nm}$.

Optical glass cuvettes of $L=1.0-, 2.0-$, and $5.0-\mathrm{mm}$ path lengths were selected to match the refractive index of toluene used in the optical glass vat. Optical path lengths of at least eight times the predicted value of $l^{*}$ for any suspension were used in order to satisfy the diffusive photon-transport approximation.

\section{B. Light scattering}

The light source for optical characterization was a Coherent Innova 70C Ar-ion laser emitting a vertically polarized beam at $488 \mathrm{~nm}$ with a beam diameter of approximately $1 \mathrm{~mm}$. Transmitted light propagated through the optical glass vat and sample, through a Glan-Thompson prism polarizer positioned perpendicular to the incident light and into the detection optics, which was focused on an area $1 \mathrm{~mm}$ in diameter on the backside of the sample. Intensity fluctuations were correlated by ALV/5000 multitau and an ALV/5000Fast correlators (ALV Laser, Langen, Germany) for temporal resolution down to $12.5 \mathrm{~ns}$.

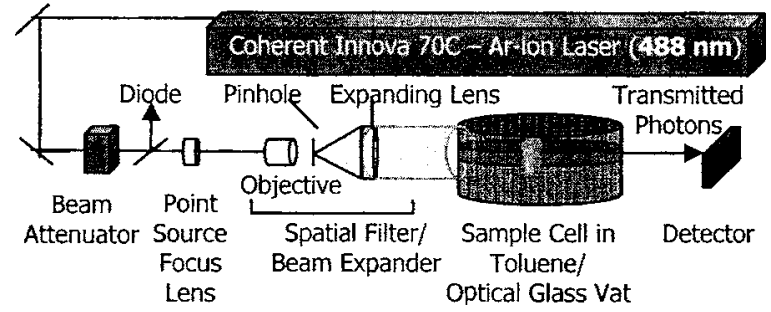

FIG. 2. Schematic drawing of optical path for DWS and DTS experiments.

The beam of the laser was expanded to a width of $38 \mathrm{~mm}$ and spatially filtered to approximate a planar source through an optical train consisting of a $60 \times$ converging lens, a 5- $\mu \mathrm{m}$ pinhole, and an expanding lens (Fig. 2). The use of a planar source in multiple-scattering optical experiments allows for simplified boundary conditions when using a diffusive photon-transport analysis. ${ }^{4}$ Opacity can be determined through transmission-mode DWS and verified with Mie scattering calculations.

A 3D Systems (Valencia, CA) SLA 250/40 stereolithography machine was used for the Windowpane experiments. The computer-driven optical train of mirrors and lenses focuses and moves the laser $(\lambda=325 \mathrm{~nm})$ to photopolymerize the suspension into the desired pattern. The $E_{\text {laser }}$ term in Eq. (1) is dependent on machine operating parameters by the relation

$$
E_{\text {laser }}=\frac{2 P h}{\pi^{2} W_{0}^{2} \nu},
$$

where $P$ is the laser power $(\mathrm{mW}), h$ is the hatch spacing $(\mathrm{cm}), W_{0}$ is the beam radius $(\mathrm{cm})$, and $\nu$ is the scanning speed $(\mathrm{cm} / \mathrm{s})$. These operating parameters, along with suspension parameters $D_{P}$ and $E_{C}$, are used to calculate the scanning speed for a specified $C_{D}$ of layers, thus linking the scattering phenomenon to the operating time.

\section{Opacity calculation}

Here we note that $l^{*}$, the mean transport free path, is inversely related to the opacity of a ceramic suspension, and for the remainder of the paper we plot data and calculations as $1 / l^{*}$ and refer to this quantity as the suspension opacity. The method described by Kaplan et al. was used to compute the opacity of monodisperse and bimodal systems with the inputs of particle radii, respective $\phi$, refractive indices of the particles and the medium, and incident wavelength. ${ }^{10}$ The addition of the structure factor contribution to the individual scattering of the particles allowed for continued analysis into higher volume fraction ranges, of particular interest to stereolithography.

The form factor $F(q)$ describes the individual scattering by each particle; the structure factor $S(q)$ is a function of particle concentration and interparticle potential. $S(q)$ provides information about the spatial distribution of the particles within the suspension. ${ }^{12,23}$ As the suspension number density $\rho$ approaches zero, $S(q)$ approaches unity, leaving only the contribution of $F(q)$ in the single-scattering case. 
The relation of $S(q)$ to opacity may be directly computed in the case of monodisperse spherical particles as

$$
\frac{1}{l^{*}}=k_{0}^{-6} d^{-4}\left[\pi \rho \int_{0}^{2 k_{0} d} I(q) q^{3} d q\right]
$$

where $\rho$ is the particle number density, $k_{0}$ is the wave vector $\left(k_{0}=2 \pi / \lambda\right), d$ is the particle diameter, and $q$ the scattering vector, is a function of the scattering angle $\theta: q$ $=2 k_{0} \sin (\theta / 2)$. For monodisperse suspensions the scattering intensity $I(q)$ is the product of the form factor and the structure factor, $F(q) S(q)$. $^{21}$

However, for a binary system of small and large particles, the need to consider cross-component interactions yields $^{21}$

$$
\begin{aligned}
I(q) \rho= & \rho_{L} S_{L L}(q) F_{L}(q)+\rho_{S} S_{S S}(q) F_{S}(q) \\
& +2 S_{L S}(q) \operatorname{Re}\left[f_{L}(q) f_{S}^{*}(q)\right]\left(\rho_{L} \rho_{S}\right)^{1 / 2},
\end{aligned}
$$

where $f(q)$ and $f^{*}(q)$ are the real and complex dimensionless far-field scattering amplitudes and $S_{i j}(q)$ are the partial structure factors to the system, representing the cross interactions between the large and small species. ${ }^{21}$ Following Kaplan et $a l$., we use an analytical solution for $S_{i j}(q)$ by Ashcroft and Langreth, which was developed using the Percus-Yevick approximation for hard-sphere suspensions. ${ }^{23,24}$

Several studies have analyzed the effects of size distribution on light scattering. ${ }^{10,25,26}$ Mean size in polydisperse systems can be characterized by a range of measures, including the arithmetic mean $\left(r_{g}\right)$ and the volume weighted average radius $\left(r_{\mathrm{vw}}\right)$. Hansen and Travis demonstrated that scattering properties of approximate log-normal distribution may be estimated by using a simple single parameter, the mean radius for scattering $\left(r_{\mathrm{eff}}\right){ }^{25}$ The value of $r_{\mathrm{eff}}$ may be computed from a fitted log-normal distribution, and is defined to be $^{26}$

$$
r_{\mathrm{eff}}=\frac{1}{G} \int_{0}^{\infty} r^{3} \pi n(r) d r
$$

where $n(r)$ is the particle radius distribution and $G$ is the geometric cross-sectional area per unit volume.

For the particle size data presented in Fig. 1(a), the curve is the least-squares fit log-normal distribution, having $r_{g}$
TABLE I. Representative particle radii $\left(r_{g}, r_{\mathrm{vw}}\right.$, and $\left.r_{\mathrm{eff}}\right)$ based on fitted log-normal particle size distributions (Fig. 1) for particles used in this experiment. The $\sigma_{g}$ value is the shape parameter used to fit the log-normal distributions.

\begin{tabular}{cccc}
\hline \hline & A16SG & A1000SG & A3500SG \\
\hline$r_{g}$ & 0.200 & 0.150 & 1.50 \\
$\sigma_{g}$ & 0.662 & 0.690 & 0.900 \\
$r_{\mathrm{vw}}$ & 0.343 & 0.261 & 1.78 \\
$r_{\mathrm{eff}}$ & 0.292 & 0.225 & 1.69 \\
\hline \hline
\end{tabular}

$=0.2 \mu \mathrm{m}$ and $\sigma_{g}=0.662 \mu \mathrm{m}$, yielding $r_{\text {eff }}=0.292 \mu \mathrm{m}$. This value of $r_{\text {eff }}$ can be used in comparison with other singlevalue descriptors in calculations of opacity. ${ }^{26}$ Table I presents calculated values of $r_{g}, \sigma_{g}, r_{\mathrm{vw}}$, and $r_{\mathrm{eff}}$ for the alumina particles used in this experiment. The results of this calculation can be compared to the log-normal distribution descriptors $r_{g}, r_{\mathrm{vw}}$, and $r_{\mathrm{eff}}$ to analyze the effect of size representation on the calculation of opacity.

\section{Planar source DWS}

To test the planar-source multiple-scattering device, planar DWS experiments were performed on monodisperse polystyrene sphere suspensions of approximately 1-2 vol \% in water. In DWS, the intensity fluctuations in multiply scattered light are measured and expressed as a normalized electric-field autocorrelation function [Eq. (8)] which includes both real and complex values of the electric field $E$,

$$
g_{1}(t)=\frac{\left\langle E(0) E^{*}(t)\right\rangle}{\left\langle|E|^{2}\right\rangle} .
$$

The experimental $g_{1}(t)$ data, extracted by procedures described by $\mathrm{Lu}$ and Solomon, enabled a least-squares fit $l^{*}$ to be determined. ${ }^{12}$ The expression of the near-exponential decay $g_{1}(t)$ as a function of $l^{*}$ was reported by Weitz and Pine ${ }^{4}$ as

$$
g_{1}(t)=\frac{\left(L / l^{*}+4 / 3\right) \sqrt{(6 t / \tau)}}{(1+8 t / 3 \tau) \sinh \left[\left(L / l^{*}\right) \sqrt{(6 t / \tau)}\right]+(4 / 3) \sqrt{(6 t / \tau)} \cosh \left[\left(L / l^{*}\right) \sqrt{(6 t / \tau)}\right]}
$$

The expression for $g_{1}(t)$ includes sample thickness $(L)$, correlation time $(t)$, Brownian diffusivity time $(\tau)$, and $l^{*}$. Equation (9) was evaluated at a number of $l^{*}$ values until the best least-squares fit to the experimental data was found and then compared to $l^{*}$ calculated by Mie scattering theory. Furthermore, the shape of the experimentally generated curve was found to match that of the calculation, so we conclude that contributions of short and long path decays from the planar source agree with the diffusion approximation of DWS. Examples of calculated $g_{1}(t)$, as well as that corresponding to the best-fit $l^{*}$ value, are shown compared with experimental data for 2-vol \% 0.260- $\mu \mathrm{m}$ polystyrene colloidal suspension in water in Fig. 3.

Transmission DWS experiments and respective Mie 


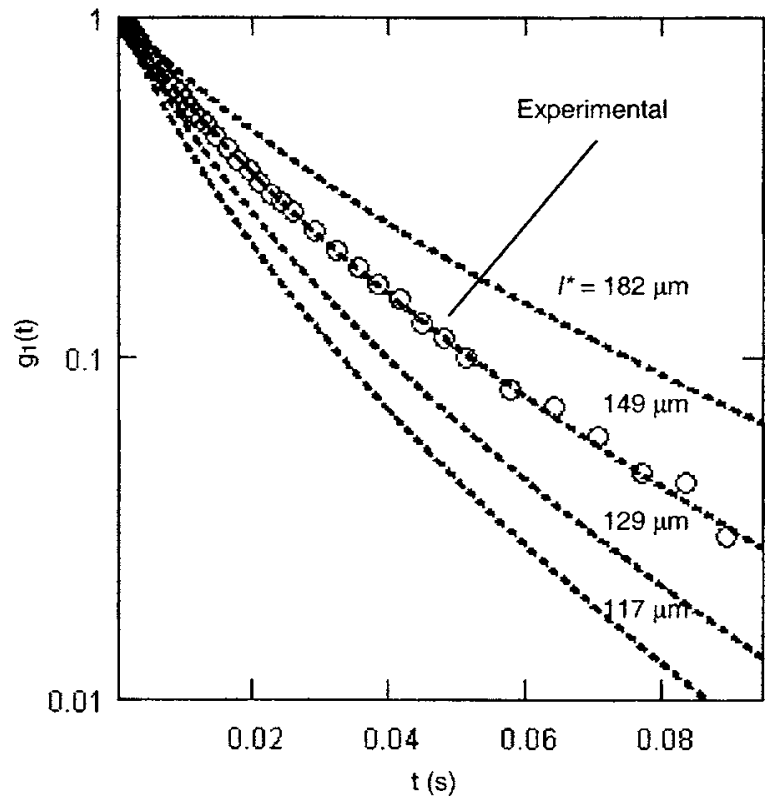

FIG. 3. Planar DWS experiment performed on monodisperse polystyrene colloidal suspension, $0.260 \mu \mathrm{m}$ in radius, at 2 vol $\%$ in water. The best least-squares fit to Eq. (9) yielded an $l^{*}$ of $149 \mu \mathrm{m}$, which is within $5 \%$ of the theoretical $l^{*}$ of $143 \mu \mathrm{m}$.

scattering calculations were performed on particles with radii that ranged from 0.090 to $1.25 \mu \mathrm{m}$. Results showed agreement within $5 \%$ error of predicted $l^{*}$ values.

\section{E. DTS experiments with aqueous polystyrene suspensions}

The DTS method allows simple determination of opacity from the transmission of light through a multiply scattering sample that satisfies the condition for diffusive photon transport $\left(L \gg l^{*}\right)$. Genack established the planar-source diffusive transmission of light, $T$, through a medium as a function of $l^{*}$ and the sample path length $L{ }^{5}$

$$
T=\frac{5 l^{*}}{3 L+4 l^{*}} \text {. }
$$

However, the differing units displayed by the diode (unitless), for the incident intensity, and the PMT (photons/s), for the scattered intensity, did not allow for the simple measurement of $T$. Rather, by computing a transmitted intensity ratio, as the average photon count detected at the PMT's divided by the incident diode reading for each sample, the appropriately scaled $T$ is compared to that of a polystyrene/water standard of known $l^{*}$ at the same measured diode reading. The standards used were the same dilute polystyrene suspensions used in the DWS experiment, with $l^{*}$ values verified by the Mie-theory-based calculation. This ratio method includes both the diode and transmitted intensities averaged over the experimental duration,

$$
\frac{T_{\text {unknown }}}{T_{\text {standard }}}=\frac{\langle\text { photon count } / \text { diode count }\rangle_{\text {unknown }}}{\langle\text { photon count } / \text { diode count }\rangle_{\text {standard }}} .
$$

The polystyrene standard thus provided a reference point for the determination of the experimental $T$ for an unknown sample. Equations (10) and (11) were then used to calculate

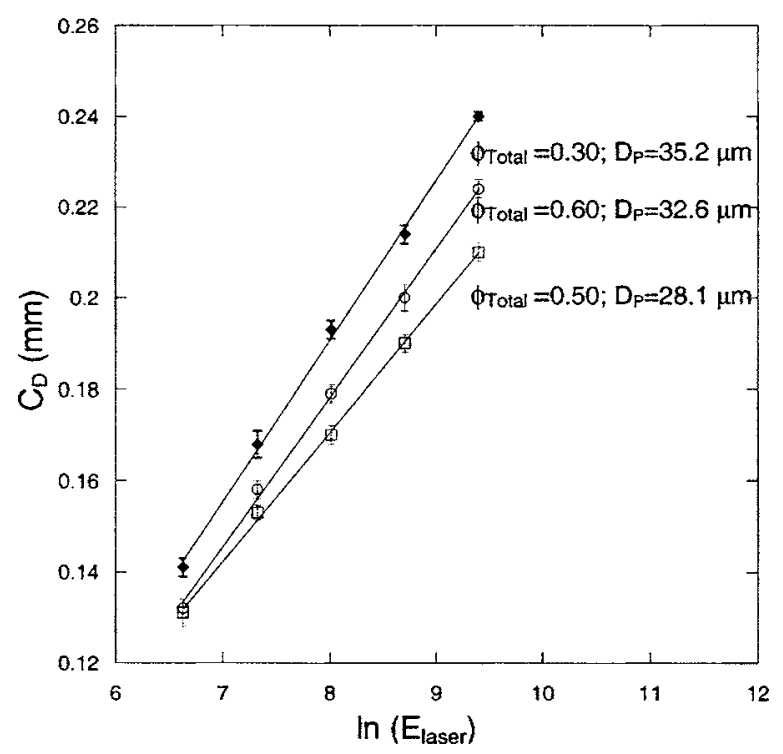

FIG. 4. Stereolithography Windowpane experimental data illustrating the linear relationship between measured $C_{D}$ and $\ln \left(E_{\text {laser }}\right)$, for $r_{g}=0.150 \mu \mathrm{m}$ alumina/monomer suspensions of $\phi_{\text {total }}=0.3,0.5$, and 0.6 . The calculated values of $D_{P}$ determined through Eq. (1) illustrate a counterintuitive increase in $D_{P}$ when $\phi_{\text {total }}$ is raised from 0.5 to 0.6 .

the $l^{*}$ value, based on the ratio of the transmitted intensity ratios. Photon counts were monitored throughout the experiment for the possibility of spurious data due to sedimentation or aggregation effects. Results from DTS experiments are reported as opacity.

\section{F. SLA Windowpanes experiments}

The diagnostic tool developed by 3D Systems for measuring stereolithography resin cure speed depends on the loglinear relationship between cure depth and exposure to the curing laser. ${ }^{2}$ Flat panes are photocured with known laser exposures, rinsed with isopropyl alcohol, and their cure depths $\left(C_{D}\right)$ are measured by micrometer. These cure depths are then plotted against the log of the respective energy dose, yielding a linear relationship with the slope defined as the resin sensitivity $\left(D_{P}\right)$ and the $x$ intercept is the critical energy $\left(E_{C}\right)$ dose required for polymerization. Figure 4 presents SLA Windowpane data for suspensions of $\phi_{\text {total }}=0.3,0.5$, and 0.6 with a photoinitiator concentration of $1 \mathrm{wt} \%$ of the monomer. The linear regressions obtained by plotting the $C_{D}$ against the energy dose of the laser yield the $D_{P}$ as the slope. The calculated values of $D_{P}$, determined through Eq. (1), are also presented, illustrating a counterintuitive increase in $D_{P}$ when $\phi_{\text {total }}$ is raised from 0.5 to 0.6 , similar to the effect seen in Fig. 7 where opacity actually decreases beyond $\phi_{\text {total }}$ $>0.4$.

\section{RESULTS}

\section{A. Polystyrene/water suspensions}

Having established a working planar source with $\lambda$ $=488 \mathrm{~nm}$ for the diffuse transmittance experiments, the calculated values of opacity were compared to experimental values in monodisperse and bimodal polystyrene/water suspensions. The volume fraction dependence of opacity for 


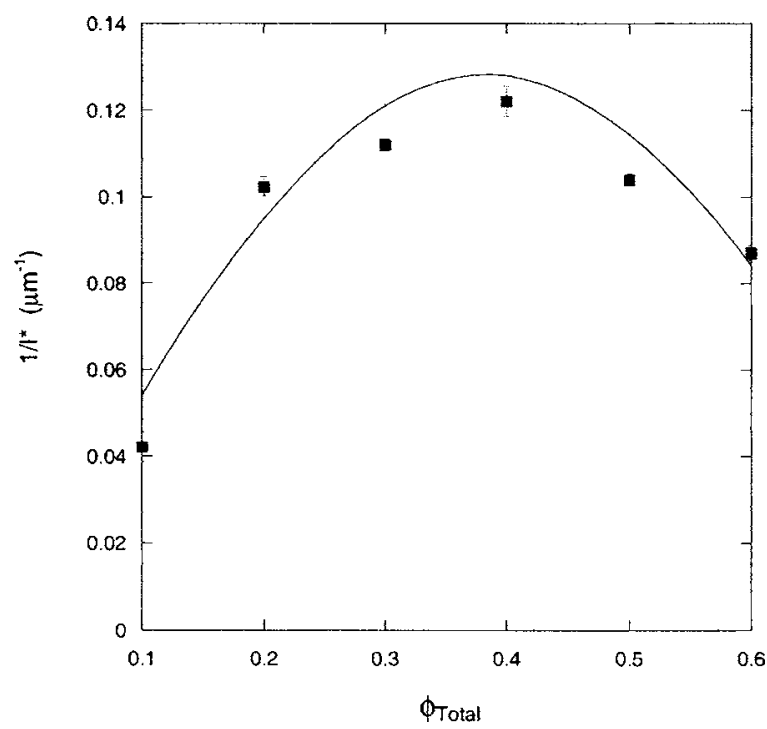

FIG. 5. Opacities measured by DTS for monodisperse polystyrene/water suspension with $\phi_{\text {total }}$ ranging from 0.1 to 0.6 , for $0.152-\mu \mathrm{m}$ radius spheres (data points). The solid curve is the calculated opacity using Eq. (5).

$0.303-\mu \mathrm{m}$ polystyrene spheres in water is reported in Fig. 5. Measurements were conducted for $0.1<\phi_{\text {total }}<0.6$. The standard error of the mean of five independent measurements is reported as error bars. These results are in agreement with Fraden and Maret in that the opacity yields a maximum at $\phi_{\text {total }} \sim 0.4$. The curve is a plot of a theoretical calculation according to Eq. (5) using the hard-sphere Percus-Yevick $S(q)$ and optical properties of polystyrene and water.

The bimodal case, with $\phi_{\text {total }}$ fixed at 0.5 , but with increasing $\phi_{\text {small }}$, is shown for 2.5 - and $0.26-\mu \mathrm{m}$ polystyrene spheres in Fig. 6. The bimodal case investigates the relative contributions of each particle size on suspension opacity. The opacity of the large particles is shown to be greater than that of the small particles, but in the bimodal range exhibits a

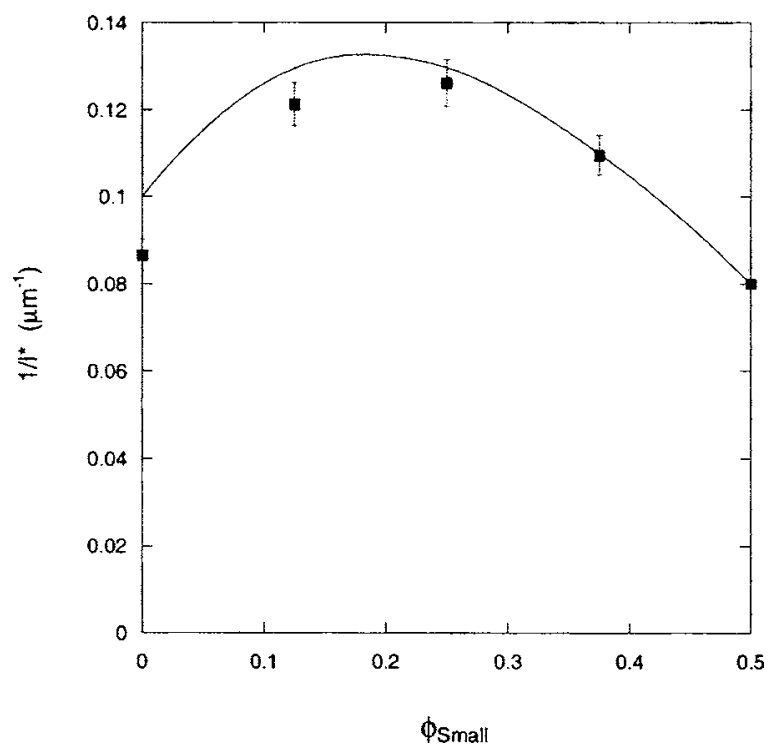

FIG. 6. Opacities measured by DTS for bimodal polystyrene/water suspensions of 1.25 - and $0.260-\mu \mathrm{m}$ radius spheres, with $\phi_{\text {total }}$ fixed at 0.5 but with increasing $\phi_{\text {small }}$. The solid curve is the calculated opacity using Eqs. (5) and (6). maximum between $0.1<\phi_{\text {small }}<0.2$. The error bars show the standard error of the mean from five independent measurements. In the polystyrene system, for particle radii of 1.25 and $0.130 \mu \mathrm{m}$, the experimentally determined values of opacity agree well with the calculations, in accordance to Eqs. (5) and (6).

Both experimental cases show good agreement with the calculated opacity values; the data demonstrate the validity of the Mie-theory-based calculation for both monodisperse and bimodal systems of uniform spherical particles.

\section{B. Alumina/monomer suspensions}

The monodisperse and bimodal experiments were repeated with alumina/monomer suspensions to test the applicability of the calculation, and the influence of the particle log-normal size distributions. Figure 7 presents the results from using particles with a $0.150-\mu \mathrm{m}$ mean radius, with $\phi_{\text {total }}$ ranging from 0.01 to 0.6 . For $\phi_{\text {total }} \leqslant 0.20$, a higher viscosity tetrafunctional acrylate monomer (SR494), with a similar refractive index $(n=1.47)$ was substituted for the difunctional monomer $(n=1.456)$ to improve suspension stability [Fig. 7(a)]. The error bars show the standard error of the mean from five independent measurements. Figure 7(b) shows results are in agreement with Garg et al. in that the opacity yields a maximum at $\phi_{\text {total }} \sim 0.4 .^{27}$ The curve, which is a plot of the theoretical calculation according to Eq. (5) using the hard-sphere Percus-Yevick $S(q)$ and optical properties of alumina $(n=1.72)$ and the respective monomers. However, the opacity values obtained in this experiment were slightly smaller than expected. Figure 7(c) presents the same results, but compared against the theoretical calculation using an effective particle refractive index of 1.70 accounting for the effect of a $\sim 5-\mathrm{nm}$ dispersant coating $(n=1.44)$. Improved agreement is observed. This indicates that the optical effect of the electrosterically stabilizing dispersant used in alumina/acrylate suspension formulation must be accounted for in optical property calculations.

For the light-scattering studies performed with alumina/ monomer suspensions, the opacity decreases with addition of the larger component, consistent with the argument made by Kaplan et al. in that the small component-imparted structure effect on the larger component particles leads to a reduction in opacity. This observation is especially relevant to the increasing $\phi_{\text {total }}$ studies, because the particle size distribution of the alumina (Fig. 1) is actually skewed toward a higher volume fraction of small particles at any particular mean size. This skewed distribution is the result of the milling process used in manufacturing the particles. This fact helps to explain the reduction in opacity in the alumina suspensions at $\phi_{\text {total }}$ approaching 0.6.

The bimodal experiment results, with $\phi_{\text {total }}$ fixed at 0.5 , but with increasing $\phi_{\text {small }}$, is shown for 1.50 - and 0.200 $-\mu \mathrm{m}$ mean radius particles in Fig. 8. The error bars show the standard error of the mean from five independent measurements. In this case, the opacity of the large particles is expected to be less than that of the small particles, and exhibits a maximum between $0.3<\phi_{\text {small }}<0.4$. The curve is a plot of the theoretical calculation according to Eqs. (5) and (6) using 

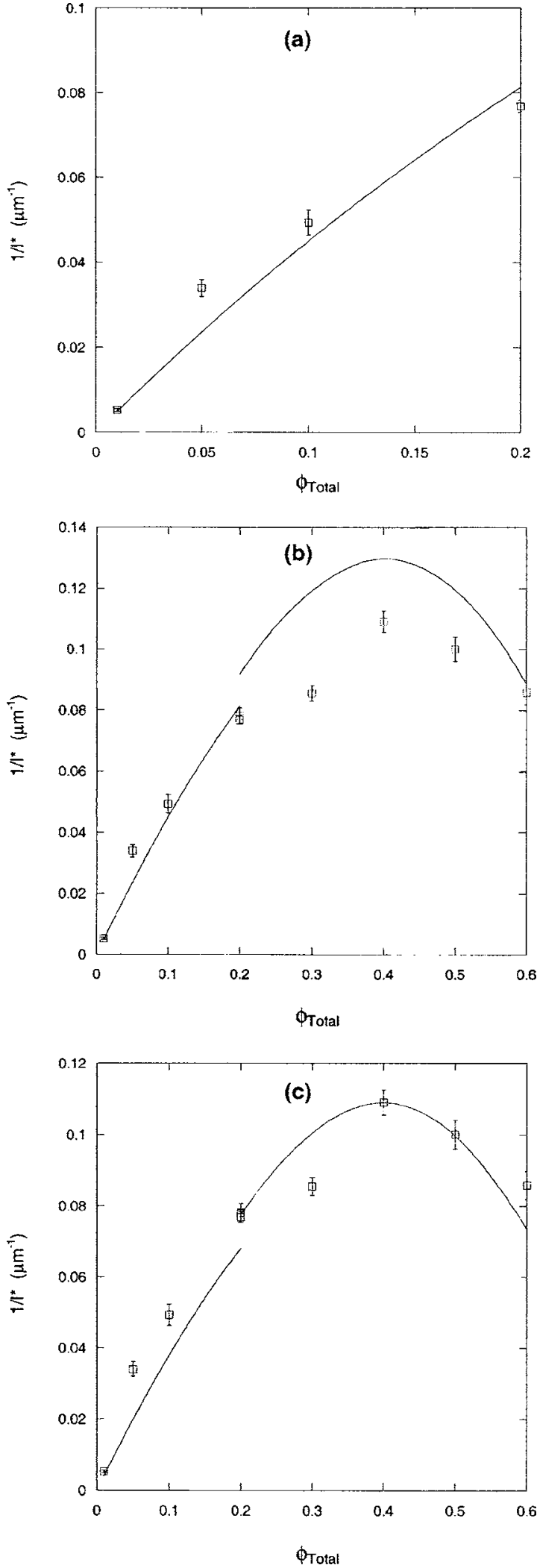

FIG. 7. Opacities measured by DTS for "monodisperse" alumina/acrylate monomer suspensions, with $\phi_{\text {total }}$ ranging from 0.05 to 0.6 for $r_{g}$ $=0.150 \mu \mathrm{m}$ particles (data points). The solid curve is the calculated opacity using Eqs. (5) and (6). A higher viscosity monomer (SR494) with a similar refractive index $(n=1.47)$ was used for $\phi_{\text {total }}<0.20$ (a), resulting in slightly different opacity calculations. Results for the monomer (SR238) with refractive index $n=1.456$ for $0.20<\phi_{\text {total }} \leqslant 0.6$ are shown in (b). (c) shows the same data as (b) with calculated opacities of particles having an effective refractive index of 1.70 .

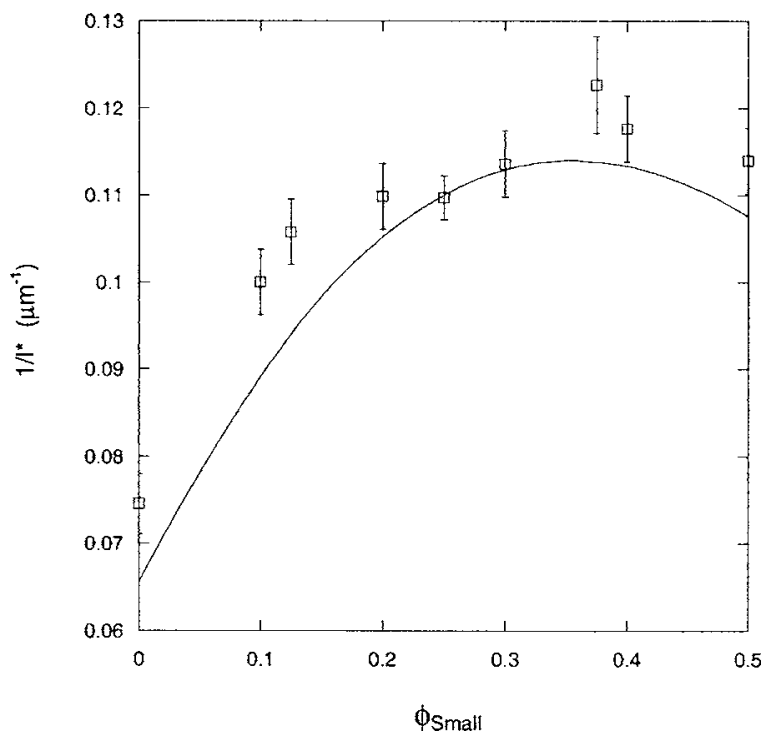

FIG. 8. Opacities measured by DTS for "bimodal" $\left(r_{g}=1.50\right.$ and $\left.0.200 \mu \mathrm{m}\right)$ alumina/acrylate monomer suspensions, with $\phi_{\text {total }}$ fixed at 0.5 but with increasing $\phi_{\text {small }}$. Good agreement was achieved in the alumina/monomer suspensions, in comparison to the calculated opacities (curve) of particles having a refractive index of 1.70 .

the hard-sphere Percus-Yevick $S(q)$ and the effective refractive index of the particles discussed above $(n=1.70)$. It is noted that with greater fractions of large particles, the data suggest a diminished optical effect by the dispersant.

The results for both the polystyrene/water and the alumina/monomer experiments are consistent with the observations of Kaplan et al. as opacity changes with increasing $\phi_{\text {small }}$. The data, showing good agreement with the calculation, indicate the significance of the partial structure factor with increasing $\phi_{\text {small }}$ in each case. In other words, opacity values show increasing deviation with increasing $\phi_{\text {small }}$ from calculations that disregard cross-component partial structure factor effects. The contribution to opacity by the small particles alone is small due to their small size relative to the incident wavelength, but their imparted structure on larger particles, through conformational limitations due to the cross-component partial structure, is significant enough to contribute to opacity. It is of interest that all these effects, originally found for monodisperse suspensions, appear to persist in commercial powders with log-normal particle size distributions.

\section{Polydispersity representation on opacity}

Figure 9 presents opacity calculations in the range of 0 $<\phi_{\text {total }}<0.6$ based on several representations of the particle size distribution of A16SG (Table I), with a mean radius of $0.200 \mu \mathrm{m}$. The log-normal fit allows for values of $r_{\mathrm{vw}}$ and $r_{\text {eff }}$ to be calculated as 0.343 and $0.292 \mu \mathrm{m}$, respectively. Opacities calculated for smallest representative size, $r_{g}=0.2$, are larger than its counterparts up to a $\phi_{\text {total }}$ of 0.4 , where the opacity begins its downturn. The larger representative values $r_{\text {eff }}$ and $r_{\mathrm{vw}}$ exhibit slightly later downturns in opacity, at $\phi_{\text {total }}$ of 0.5 and 0.55 , respectively. Experimental results for 


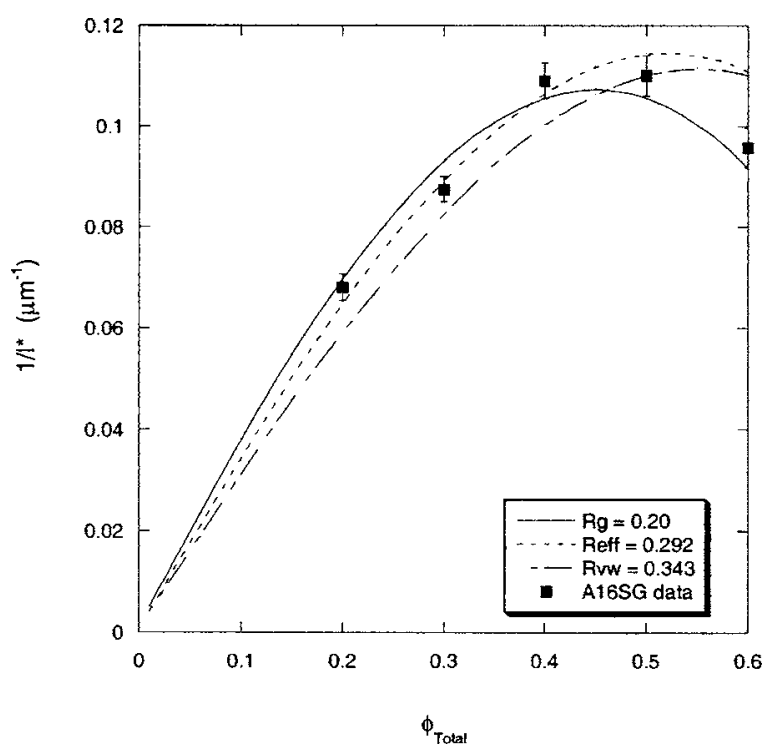

FIG. 9. Calculated opacities as function of $\phi_{\text {total }}$ based on different representations of log-normal particle size distribution of Al6SG alumina powder [Fig. 1(a)]. Results from mean values $r_{g}, r_{\mathrm{eff}}$, and $r_{\mathrm{vw}}$ are compared against the experimental data.

the A16SG powder $\left(r_{g}=0.2 \mu \mathrm{m}\right)$ in HDDA $(n=1.456)$ between $0.2<\phi_{\text {total }}<0.6$ agree best with the opacity calculation performed with the $r_{g}$ value.

Although the theory does not account for the modestly aspherical shape or the log-normal size distributions of the alumina particles, the demonstrated agreement of opacity in these results with theory indicates that by using a mean particle size and assuming spherical geometry of the moderately aspherical particles in the calculation can yield reasonable estimates of opacity.

\section{Comparing $I^{t}$ and $D_{P}$ in ceramic stereolithography}

With the addition of photoinitiator, at concentrations of $0.5-, 1-, 2-$, and 5-wt \% monomer, $D_{P}$ values were determined by building Windowpane diagnostic parts by means of SLA. Figures 10(a)-10(c) plot measured $D_{P}$ values against experimental variables $\phi_{\mathrm{Small}}, c_{\mathrm{PI}}$, and $l^{*}$, respectively. The lines are intended to guide the eye. These figures illustrate that $D_{P}$ is not simply a function of variables $\phi, c_{\mathrm{PI}}$, or $l^{*}$. The data set in Fig. 10(a) corresponds to "bimodal" $\left(r_{g}=0.200\right.$ and $1.50 \mu \mathrm{m}$ ) alumina suspensions of increasing $\phi_{\text {small }}$ at a fixed $\phi_{\text {total }}$ of 0.5. Figure 10(b) shows $D_{P}$ data from bimodal and "monodisperse" scattering experiments as a function of $c_{\mathrm{PI}}$ at fixed $l^{*}$ values. Figure 10(c) compares $D_{P}$ and $l^{*}$ values for both bimodal cases and both monodisperse cases at fixed $c_{\mathrm{PI}}$ values. These figures exhibit a convergence of $D_{P}$ values when approaching the strongest-scattering cases (small $l^{*}$ ), indicating decreasing effect of photoinitiator absorption on the resultant $D_{P}$ in these scattering-dominated regimes. However, these figures illustrate that $D_{P}$ is not simply a function of variables $\phi, c_{\mathrm{PI}}$, or $l^{*}$, pointing to the need for an allinclusive model. The relationship between $l^{*}$ and $D_{P}$ of the alumina suspensions are better understood by plotting data in accordance to Eq. (3), while taking into account the BeerLambert absorption behavior between the particles in the suspension at various photoinitiator concentrations (Fig.
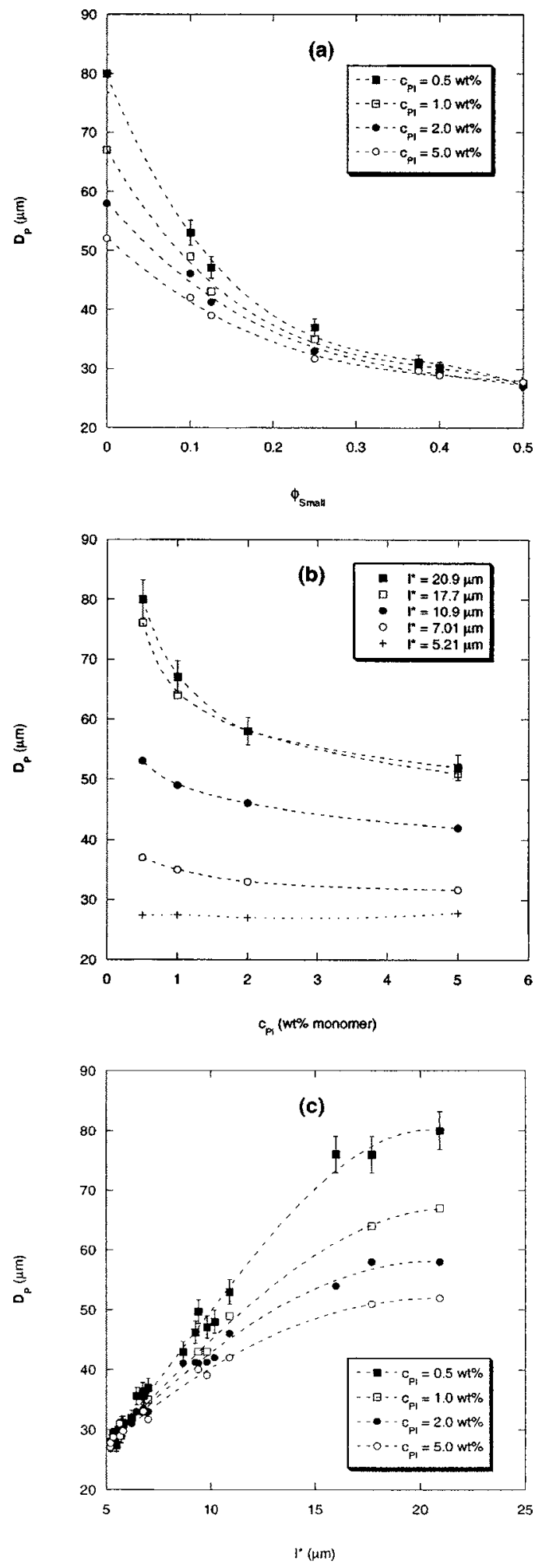

FIG. 10. (a)-(c) plot measured $D_{P}$ values against experimental variables $\phi_{\text {Small }}, c_{\mathrm{PI}}$, and $l^{*}$, respectively. The lines are intended to guide the eye. These figures illustrate that $D_{P}$ is not simply a function of variables $\phi, c_{\mathrm{PI}}$, or $l^{*}$. The data set in (a) corresponds to "bimodal" $\left(r_{g}=0.200\right.$ and $\left.1.50 \mu \mathrm{m}\right)$ alumina suspensions of increasing $\phi_{\text {small }}$ at a fixed $\phi_{\text {total }}$ of 0.5 . (b) shows $D_{P}$ data from both bimodal $\left(r_{g}=0.200\right.$ and $\left.1.50 \mu \mathrm{m}\right)$ and "monodisperse" $\left(r_{g}\right.$ $=0.200)$ scattering experiments as a function of $c_{\mathrm{PI}}$ at fixed $l^{*}$ values. (c) compares $D_{P}$ and $l^{*}$ values for both bimodal cases $\left(r_{g}=0.150\right.$ and $1.50 \mu \mathrm{m}$ and $r_{g}=0.200$ and $\left.1.50 \mu \mathrm{m}\right)$ and both monodisperse cases $\left(r_{g}=0.150\right.$ and $\left.r_{g}=0.200\right)$ at fixed $c_{\mathrm{PI}}$ values. These figures exhibit a convergence of $D_{P}$ values when approaching the strongest-scattering cases (small $l^{*}$ ), indicating decreasing effect of photoinitiator absorption on the resultant $D_{P}$ in these scattering-dominated regimes. 


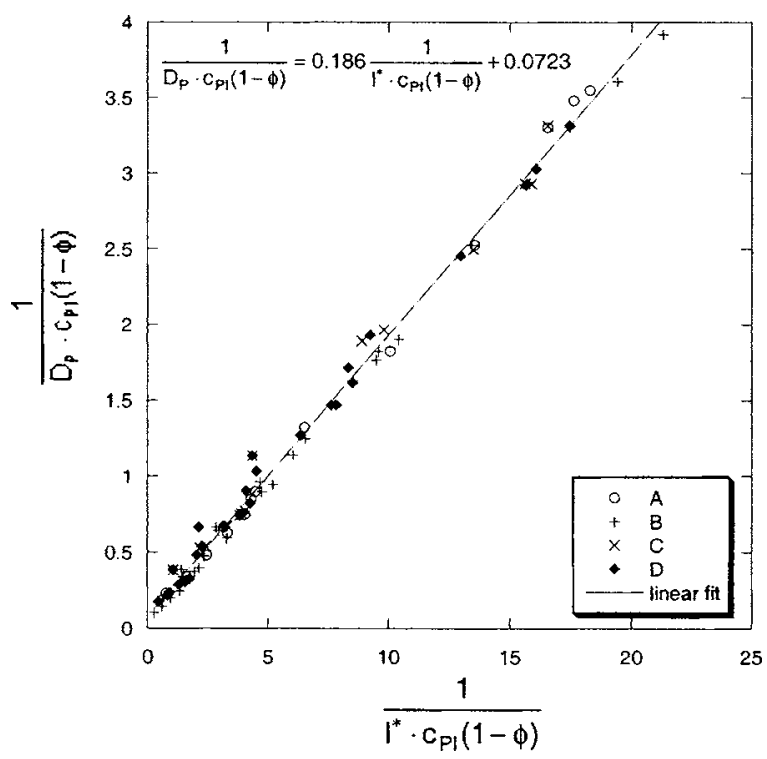

FIG. 11. Relating the $D_{P}$ data from Windowpanes to calculated values of $l^{*}$ for four types of alumina/acrylate suspensions; monodisperse suspension $\phi_{\text {total }}$ ranged from 0.1 to 0.6 , and bimodal suspensions held a fixed $\phi_{\text {total }}$ of 0.5 with increasing $\phi_{\text {small }}$. Data sets A and B correspond to $l^{*}$ and $D_{P}$ measurements of "monodisperse" $\left(r_{g}=0.150\right.$ and $\left.0.200 \mu \mathrm{m}\right)$ alumina suspensions at $0.1<\phi_{\text {total }}<0.6$, respectively. Data sets $\mathrm{C}$ and D correspond to "bimodal" $\left(r_{g}=0.15\right.$ and $1.5 \mu \mathrm{m}$ and $r_{g}=0.20$ and $\left.1.5 \mu \mathrm{m}\right)$ alumina suspensions of increasing $\phi_{\text {small }}$ at a fixed $\phi_{\text {total }}$ of 0.5 . The linear fit shows that $D_{P}$ is shown to be approximately five times the distance of photon-transport mean free path $\left(l^{*}\right)$, with an intercept value, or the photoinitator molar extinction coefficient, of $0.0723 \mathrm{M}^{-1} \mu \mathrm{m}^{-1}$.

11). ${ }^{27}$ The $l^{*}$ values are calculated using the effective refractive index value of 1.70 and an incident wavelength of $325 \mathrm{~nm}$, consistent with the SLA laser. The empirical constant $A$ is shown to be 0.1856 , indicating that $D_{P}$ is approximately five times the distance of photon-transport mean free path, in agreement with Garg et al. ${ }^{27}$ Furthermore, the results indicate a photoinitiator molar extinction coefficient of $0.0723 \mathrm{M}^{-1} \mu \mathrm{m}^{-1}$, in very good agreement with the value of $0.0700 \mathrm{M}^{-1} \mu \mathrm{m}^{-1}$ at the wavelength provided by the manufacturer, providing further confirmation of the success of Eq. (3).

\section{DISCUSSION}

The results obtained here extend the understanding of structural contributions in multiple scattering to suspensions containing particles of moderately aspherical shape and lognormal particle size distribution in agreement with the theories posed by Fraden and Maret and by Kaplan et al. Equation (3) fits the data (Fig. 11) quite well, with an $R^{2}$ statistic of 0.997 . The success of this equation is further supported by the excellent agreement between the extrapolated and actual values of the molar extinction coefficient indicative of the absorption properties of the photoinitiator. Applying this tool to ceramic stereolithography will allow for process optimization tailored to ceramic suspensions of interest.

Results from the alumina/monomer experiments, which demonstrate a decrease in opacity when $\phi_{\text {total }}>0.5$, are particularly pertinent to ceramic stereolithography. Increasing $\phi_{\text {total }}$ of the ceramic suspensions would increase green body strength, while potentially providing an advantage in terms of opacity, and thus, processing speed. However, the increase in $\phi_{\text {total }}$ is likely to increase the suspension viscosity, leading to significantly longer recoat times. However, formulating a ceramic stereolithography suspension from bimodally sized particles has benefits in terms of viscosity without much penalty in terms of opacity (Fig. 8). Note that in Fig. 8, there is an insignificant difference in opacity between suspensions of $0.25<\phi_{\text {small }}<0.5$. A reduction of suspension viscosity achieved by changing the particle size distribution from monodisperse to bimodal is known as the Farris effect. ${ }^{28}$ This discussion demonstrates the utility of incorporating the partial structure factor model into the computational tool.

\section{CONCLUSIONS}

A Mie-theory-based computational method of obtaining opacity, including partial structure factor contributions for bimodal suspensions, is found to be applicable to predicting opacity of suspensions containing nonabsorbing alumina particles of moderately aspherical size and a log-normal size distribution.

A general, quantitative relationship between the photontransport mean free path $\left(l^{*}\right)$ and resin sensitivity $\left(D_{P}\right)$ in multiple-scattering alumina/acrylate suspensions formulated for ceramic stereolithography is proposed and experimentally demonstrated.

\section{ACKNOWLEDGMENTS}

This research was funded by the Office of Naval Research. It is a pleasure to acknowledge the assistance of A. Mohraz in aspects of this study.

${ }^{1}$ M. L. Griffith and J. W. Halloran, J. Am. Ceram. Soc. 79, 2601 (1996). ${ }^{2} \mathrm{P}$. F. Jacobs, Stereolithography and Other RP \& M Technologies (ASME, New York, 1996).

${ }^{3}$ R. Garg, R. Prud'homme, I. Aksay, F. Liu, and R. Alfano, J. Mater. Res. 13, 3463 (1998).

${ }^{4}$ W. Brown, Dynamic Light Scattering: The Method and Some Applications, edited by D. Weitz and D. Pine (Clarendon, Oxford, 1993).

${ }^{5}$ A. Z. Genack, Phys. Rev. Lett. 58, 2043 (1987).

${ }^{6}$ M. U. Vera and D. J. Durian, Phys. Rev. E 53, 3215 (1996).

${ }^{7}$ P. M. Saulnier, M. P. Zinkin, and G. H. Watson, Phys. Rev. B 42, 2621 (1990).

${ }^{8}$ D. J. Pine, D. A. Weitz, G. Maret, P. E. Wolf, E. Herbolzheimer, and P. M. Chaikin, in Scattering and Localization of Classical Waves in Random Media, edited by P. Sheng (World Scientific, Singapore, 1990), pp. 312371

${ }^{9}$ D. J. Durian, Phys. Rev. E 51, 3350 (1995).

${ }^{10}$ K. F. Seefeldt, Ph.D. thesis, University of Michigan, 2002.

${ }^{11}$ C. F. Bohren and D. R. Huffman, Absorption and Scattering of Light by Small Particles (Wiley, New York, 1983), p. 126.

${ }^{12}$ Q. Lu and M. J. Solomon, Phys. Rev. E 66, 061504/1 (2002).

${ }^{13}$ H. M. Wyss, J. Innerlohinger, L. P. Meier, L. J. Gauckler, and O. Glatter, J. Colloid Interface Sci. 271, 388 (2004).

${ }^{14}$ M. U. Vera and D. J. Durian, Phys. Rev. E 53, 3215 (1996).

${ }^{15}$ R. Pecora, Dynamic Light Scattering: Applications of Photon Correlation Spectroscopy (Plenum, New York, 1985).

${ }^{16}$ H. M. Wyss, S. Romer, F. Scheffold, P. Schurtenberger, and L. J. Gauckler, J. Colloid Interface Sci. 241, 89 (2001).

${ }^{17}$ F. C. MacKintosh and J. Sajeev, Phys. Rev. B 40, 2383 (1989).

${ }^{18}$ S. Fraden and G. Maret, Phys. Rev. Lett. 65, 512 (1990).

${ }^{19}$ V. Twersky, J. Opt. Soc. Am. 65, 524 (1975).

${ }^{20}$ A. Ishimaru and Y. Kuga, J. Opt. Soc. Am. 72, 1317 (1982).

${ }^{21}$ P. Kaplan, A. Yodh, and D. Pine, Phys. Rev. Lett. 68, 393 (1993). 
${ }^{22}$ K. F. Seefeldt and M. J. Solomon, Phys. Rev. E 67, 050402(R) (2003).

${ }^{23}$ N. Ashcroft and D. Langreth, Phys. Rev. 156, 685 (1967); 166, 934(E) (1968).

${ }^{24}$ J. K. Percus and G. J. Yevick, Phys. Rev. 110, 1 (1958).

${ }^{25}$ J. E. Hansen and L. D. Travis, Space Sci. Rev. 16, 527 (1974).

${ }^{26}$ M. I. Mishchenko and L. D. Travis, Appl. Opt. 33, 7206 (1994).
${ }^{27}$ R. Garg, R. Prud'homme, I. Aksay, F. Liu, and R. Alfano, J. Opt. Soc. Am. A 15, 932 (1998).

${ }^{28} \mathrm{R}$. Larson, The Structure and Rheology of Complex Fluids (Oxford University Press, New York, 1999), p. 267.

${ }^{29}$ C. Sun and X. Zhang, J. Appl. Phys. 92, 4796 (2002).

${ }^{30}$ C. Sun and X. Zhang, Sens. Actuators, A 101, 364 (2002). 